\title{
Shear-strain-mediated large nonvolatile tuning of ferromagnetic resonance by an electric field in multiferroic heterostructures
}

\author{
Ming Zheng $\mathbb{D}^{1,2}$, Takamasa Usami ${ }^{2}$ and Tomoyasu Taniyama (D) ${ }^{2,3}$
}

\begin{abstract}
Controlling magnetism by an electric field is of critical importance for the future development of ultralow-power electronic and spintronic devices. Progress has been made in electrically driven nonvolatile tuning of magnetic states in multiferroic heterostructures for the information storage industry, which is exclusively attributed to the ferroelectricpolarization-switching-induced interfacial charge effect or nonlinear lattice strain effect. Here, we demonstrate that a hitherto unappreciated shear strain in the ferroelectric $0.7 \mathrm{~Pb}\left(\mathrm{Mg}_{1 / 3} \mathrm{Nb}_{2 / 3}\right) \mathrm{O}_{3}-0.3 \mathrm{PbTiO}_{3}$ substrate triggered by an electric field can be adopted to obtain robust nonvolatile control of the ferromagnetic resonance in an elastically coupled epitaxial $\mathrm{Fe}_{70} \mathrm{Rh}_{30}$ thin film. The disappearance of the resonance peak in a low-field-sweeping mode and the large resonance field shift of 111 Oe upon polarization switching demonstrate a strong shear-strain-mediated magnetoelectric coupling effect. In particular, in situ Kerr measurement identifies that the nonvolatile magnetic switching purely originates from electric-field-induced $109^{\circ}$ ferroelastic domain switching rather than from $71^{\circ} / 180^{\circ}$ ferroelectric domain switching even without the assistance of a magnetic field. This discovery illustrates the role of shear strain in achieving electrically tunable nonvolatile modulation of dynamic magnetic properties, and favors the design of future energy-efficient magnetoelectric microwave devices.
\end{abstract}

\section{Introduction}

The integration of magnetic thin films (e.g., metals/ alloys, ferrites, manganites, and dilute magnetic semiconductors) with perovskite ferroelectrics provides a new avenue for modifying magnetism by electric fields (instead of magnetic fields and electric currents) through a strong magnetoelectric (ME) coupling effect, which shows promise for application in high-speed, lightweight, and ultralow-power tunable electronic and spintronic devi$\operatorname{ces}^{1-17}$. Recent efforts have been made toward electrically controlling a number of different magnetic effects in these multiferroic heterostructures, such as the tunneling anisotropic magnetoresistance ${ }^{2}$, topological anomalous Hall

\footnotetext{
Correspondence: Ming Zheng (zheng-m@hotmail.com) or

Tomoyasu Taniyama (taniyama.tomo@nagoya-u.jp)

${ }^{1}$ School of Materials Science and Physics, China University of Mining and Technology, 221116 Xuzhou, China

${ }^{2}$ Department of Physics, Nagoya University, Furo-cho, Chikusa-ku, Nagoya 464-8602, Japan

Full list of author information is available at the end of the article
}

effect $^{3}$, magnetic domain wall motion ${ }^{6}$, in-plane/perpendicular magnetic anisotropy ${ }^{7,8}$, exchange bias ${ }^{10}$, magnetic phase transition ${ }^{3,4,14}$ and ferromagnetic resonance $(\mathrm{FMR})^{11}$, as well as toward nonvolatile tuning of magnetic states $^{12,18}$. From a practical device application perspective, it is highly desirable to tailor magnetic performances in a nonvolatile way. Namely, the magnetic states can be maintained after turning off the control electric field. Originally, the most common method was to utilize the remnant electric polarization of ferroelectrics to create a thin surface charge density at the interface of magnetic/ ferroelectric composites (i.e., the ferroelectric field effect), which can offer nonvolatile switching of magnetic properties in multiferroic heterostructures via a charge-driven ME coupling effect ${ }^{18,19}$. However, the electrically assisted interfacial charge effect can only be experimentally detected in ultrathin atomically flat ferromagnetic layers or at low temperatures ${ }^{19}$, because of the infinitesimal screening length (a few unit cells) and/or large charge

\section{(c) The Author(s) 2021}

(c) (i) Open Access This article is licensed under a Creative Commons Attribution 4.0 International License, which permits use, sharing, adaptation, distribution and reproduction cc) in any medium or format, as long as you give appropriate credit to the original author(s) and the source, provide a link to the Creative Commons license, and indicate if changes were made. The images or other third party material in this article are included in the article's Creative Commons license, unless indicated otherwise in a credit line to the material. If material is not included in the article's Creative Commons license and your intended use is not permitted by statutory regulation or exceeds the permitted use, you will need to obtain permission directly from the copyright holder. To view a copy of this license, visit http://creativecommons.org/licenses/by/4.0/. 
carrier density in the channel films, which hinders the field-effect transistor applications. Later, the technique was extended to employ nonlinear lattice strain effects of ferroelectric $\mathrm{Pb}\left(\mathrm{Mg}_{1 / 3} \mathrm{Nb}_{2 / 3}\right) \mathrm{O}_{3}-\mathrm{PbTiO}_{3} \quad$ (PMN-PT) single-crystal substrates. Aside from the stable upward and downward remnant polarization states, a metastable in-plane polarization state appears during $180^{\circ}$ ferroelectric domain reorientation in the PMN-PT substrates $^{20,21}$. Therefore, nonvolatile tuning can be realized at room temperature using two stable residual strain states through domain-engineered ferroelastic switching (i.e., by rotating the ferroelectric polarization between the out-of-plane and in-plane directions). According to this design principle, nonvolatile control of magnetic, electrical, and optical properties has been proven in some functional thin films, including ferromagnetic $\mathrm{Fe}_{40} \mathrm{Pt}_{60}$ (ref. ${ }^{12}$ ), ferrimagnetic $\mathrm{Fe}_{3} \mathrm{O}_{4}$ (ref. ${ }^{22}$ ), ferroelectric luminescent $\left(\mathrm{Ba}_{0.85} \mathrm{Ca}_{0.15}\right)_{0.998} \operatorname{Pr}_{0.002} \mathrm{Ti}_{0.9} \mathrm{Zr}_{0.1} \mathrm{O}_{3}$ (ref. ${ }^{23}$ ), multiferroic $\mathrm{Bi}_{0.95} \mathrm{Mn}_{0.05} \mathrm{FeO}_{3}$ (ref. ${ }^{24}$ ), Mott insulating $\mathrm{VO}_{x}{ }^{25-27}$, and $\mathrm{NdNiO}_{3}$ (ref. ${ }^{28}$ ), ferromagnetic metallic $\mathrm{SrRuO}_{3}$ (ref. ${ }^{29}$ ), and colossal magnetoresistive $R_{1-x} A_{x} \mathrm{MnO}_{3}(R=\mathrm{La}, \mathrm{Nd} ; A=\mathrm{Ca}, \mathrm{Sr})^{30-33}$, by depositing them onto weakly clamped PMN-PT substrates and selectively controlling the polarization switching pathway. Despite intense efforts dedicated to nonvolatile switching of lattice-related functionalities in PMN-PT-based heterostructures using the domain-engineered nonlinear strain effect, nonvolatile ME coupling mediated by electric-field-induced shear strain has remained elusive.

In this work, we report epitaxial integration of $\mathrm{Fe}_{70} \mathrm{Rh}_{30}$ (FeRh) thin films with ferroelectric PMN-PT single crystals and investigate the nonvolatile electric field effect on their FMR properties at room temperature. FeRh is chosen due to its large magnetic moment and high Curie temperature $\left(>700^{\circ} \mathrm{C}\right)$ without a magnetic phase transition from antiferromagnetic to ferromagnetic order ${ }^{34,35}$. Our results verify that the $109^{\circ}$ ferroelastic domain switching of the PMN-PT substrate enables the presence of in-plane shear strain, which, in turn, efficiently manipulates the magnetization and FMR of the FeRh film in a nonvolatile manner by adjusting the competition between the magnetocrystalline anisotropy energy and the uniaxial anisotropy energy. This work represents a new development that shear-strain-mediated ME coupling can be considered for building advanced ultralow-power ME memory devices.

\section{Materials and methods}

$\mathrm{A} \mathrm{SrTiO}_{3}(\mathrm{STO})$ thin layer $(10 \mathrm{~nm})$ was first grown on a (001)-cut PMN-PT commercial single-crystalline wafer as a buffer layer, using a pulsed laser deposition technique to avoid interfacial diffusion, and chemical reaction between chemically active FeRh and PMN-PT. Subsequently, a FeRh thin film $(30 \mathrm{~nm})$ was fabricated on the STObuffered PMN-PT substrate using molecular beam epitaxy with a base pressure $<10^{-10}$ Torr, followed by growing $3 \mathrm{~nm}$ of $\mathrm{Rh}$ as a capping layer to prevent oxidation. A Au layer $(100 \mathrm{~nm})$ was coated on the backside of the PMN-PT substrate as a bottom electrode.

The crystal structure and epitaxial nature of the film were characterized using a high-resolution X-ray diffractometer (HR-XRD, Bruker D8 Discover) equipped with a $\mathrm{Ge}(220)$ monochromator and $\mathrm{Cu} K_{\alpha 1}$ radiation. Ferroelectric polarization switching of the PMN-PT substrate was carried out by imposing an electric field across the FeRh/PMN-PT structure through the metallic Rh top electrode and the Au bottom electrode, using a Keithley $6517 \mathrm{~B}$ voltage source. The FMR spectra were recorded on a vector network analyzer (VNA, E5071C ENA Series, Agilent Technologies) with the sample positioned face down on a broadband coplanar waveguide. The VNA transmission parameter $S_{21}$, i.e., the ratio of the outgoing microwave signal at port 2 to the incident microwave signal at port 1 , was measured over a frequency range of up to $20 \mathrm{GHz}$ with magnetic fields along the PMN-PT [100] direction. Magnetic hysteresis loops were collected using a vibrating sample magnetometer (Lakeshore $7407 \mathrm{~N}$ ). All measurements were performed at room temperature.

\section{Results and discussions}

The XRD out-of-plane $\theta-2 \theta$ scan spectrum of the FeRh/ PMN-PT heterostructure (see Fig. 1a) reveals that the FeRh film is highly (001)-oriented without any impurity phases or other orientations. The rocking curve of the FeRh (002) peak in Fig. 1b exhibits a full-width at halfmaximum of $0.98^{\circ}$, indicating the superior crystallinity of the film. XRD $\phi$ scans around the (101) reflection peaks of the FeRh film and the PMN-PT substrate were conducted to establish the in-plane crystallographic orientation of the film with respect to the substrate. As displayed in Fig. 1c, two sets of fourfold symmetric reflection peaks are observed, implying good heteroepitaxy. The epitaxial relationship of the film with respect to the substrate is described as $(001)_{\mathrm{FeRh}} \|(001)_{\mathrm{PMN}-\mathrm{PT}}$ and $[110]_{\mathrm{FeRh}} \|$ $[010]_{\text {PMN-PT. }}$. We then obtained the in-plane lattice arrangement for the cubic FeRh unit cell on a pseudocubic PMN-PT substrate, as shown in Fig. 1d. A good epitaxial heterointerface is crucial for electric control of the magnetism in multiferroic heterostructures that seeks to exploit strong interfacial mechanical strain coupling.

To gain insight into the ME coupling in the FeRh/PMNPT system, we employed longitudinal magneto-optical Kerr effect (MOKE) measurements with magnetic fields applied along the [100] direction of the PMN-PT substrate to record magnetic switching as a function of the applied electric field $(E)$, as shown in Fig. 2a. The Rh capping layer is thin enough to be transparent to the laser beam with a wavelength of $\lambda=660 \mathrm{~nm}$. As seen in the inset of Fig. 2b, the Kerr hysteresis loop signifies a typical ferromagnetic 

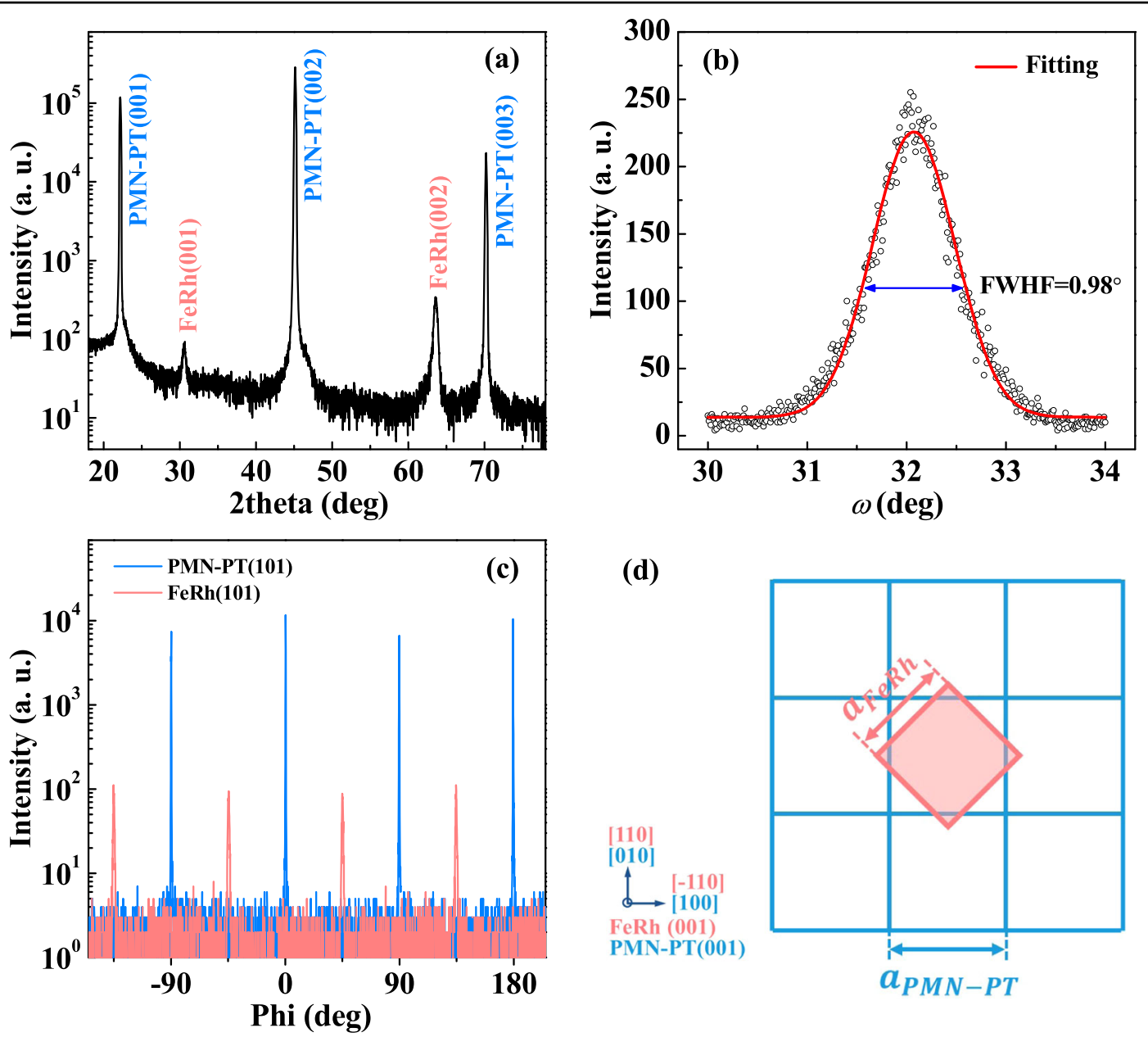

Fig. 1 FeRh/PMN-PT heteroepitaxial structure. a XRD out-of-plane $\theta-2 \theta$ scan spectrum of the FeRh/PMN-PT heterostructure. $\mathbf{b}$ Rocking curve of the FeRh (002) peak. c XRD $\phi$ scans around the FeRh (101) and PMN-PT (101) reflection peaks. $\mathbf{d}$ Schematic of the epitaxial relationship between FeRh and PMN-PT.

nature of the FeRh film. Since the Kerr signal is proportional to the in-plane remnant magnetization $M_{r}$ of the film, the electric field control of $M_{r}$ can be manifested by the relative evolution of the Kerr signal in Fig. 2b. A sharp variation in the remnant magnetization of the FeRh film occurs upon polarization switching of the PMN-PT substrate, indicating an effective cross-coupling between two ferroic (ferromagnetic and ferroelectric) order parameters. The clear loop-like hysteretic $M_{r}-E$ curve proves a reversible and nonvolatile converse ME effect, favorable for ultralow-power nonvolatile magnetic information encoding. It is worth noting that the Kerr signal is gradually reduced with increasing electric field after the field exceeds the coercive field $\left(E_{C} \sim 1.4 \mathrm{kV} / \mathrm{cm}\right)$ of PMN-PT regardless of the bias voltage polarity, which confirms the contribution of the volatile butterfly-like behavior to the nonvolatile loop-like ME coupling. Using a simple mathematical transformation of the $M_{r}-E$ data $^{36,37}$, we deduce the symmetric butterfly-like $\left(f_{\text {sym }}=[M(-E)+M(+E)] / 2\right)$ and antisymmetric loop-like $\quad\left(f_{\text {asym }}=[M(-E)-M(+E)] / 2\right)$ parts of the $M_{r}-E$ curve, shown in Fig. 2c, d, respectively. It is well known that the electric-field-induced strainmediated and charge-mediated coupling mechanisms in multiferroic heterostructures can plausibly account for the butterfly-like and loop-like converse ME effects, respectively ${ }^{19,38}$. Considering the infinitesimal screening length (a few unit cells) of the electron in the FeRh film ${ }^{4}$ and its separation from PMN-PT by the STO buffer layer, the electrostatic charge effect is negligible in our 30-nm thick FeRh film. Fortunately, the electrically driven ferroelastic strain effect due to non- $180^{\circ}$ domain switching in PMNPT substrates has been widely exploited to realize nonvolatile loop-like electric field manipulation of the magnetic, electrical, and optical properties of overlying functional thin films ${ }^{12,22-33}$. It is thus anticipated that a close relationship exists between the nonvolatile magnetic response of the FeRh film and the domain switching dynamics of the PMN-PT substrate. 

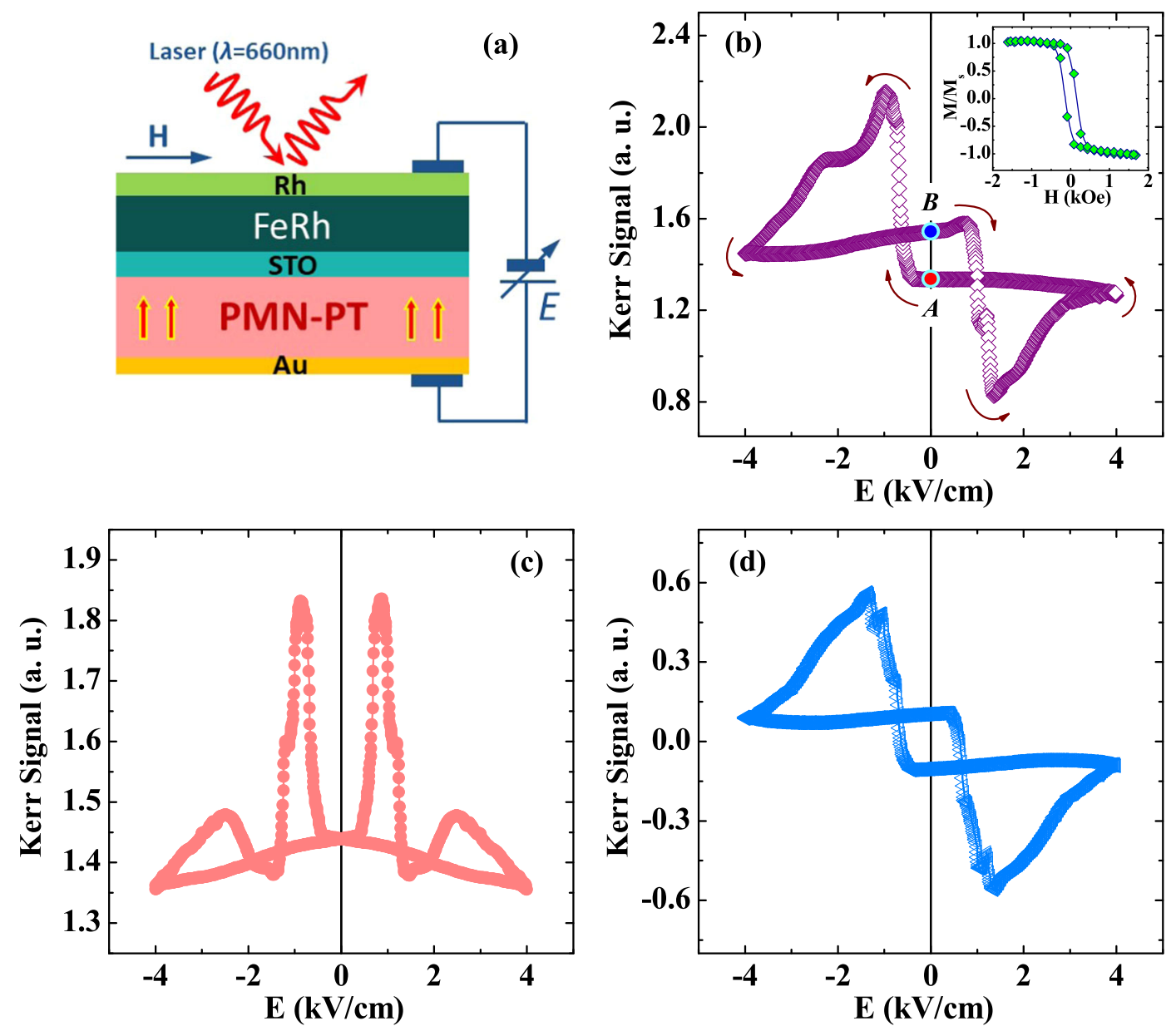

Fig. 2 Nonvolatile magnetic switching driven by an electric field in FeRh/PMN-PT heterostructure. a Schematic of the experimental setup for in situ measurement of magnetic switching of the FeRh/PMN-PT heterostructure under an electric field using longitudinal MOKE measurement. b Magnetoelectric hysteresis curve showing the dependence of the remnant magnetization of the system on the electric field, from which $\mathbf{c}$ the volatile butterfly-like and $\mathbf{d}$ the nonvolatile loop-like parts are separated. The inset in $\mathbf{b}$ shows the normalized in-plane Kerr hysteresis loop of the structure.

In the rhombohedral PMN-PT single-crystal substrate, the eight spontaneous polarization vectors point in the body-diagonal directions of the pseudocubic unit cell in the virgin state with four structural domains $\left(P_{1}, P_{2}, P_{3}\right.$, and $\left.P_{4}\right)$. Upon applying a large positive field $E=+4 \mathrm{kV} / \mathrm{cm}$ $\left[E>E_{C(\mathrm{PMN}-\mathrm{PT})}\right]$, PMN-PT is fully positively polarized, and its polarization vector aligns along $P_{1}^{+}$(hereafter referred to as the $P_{r}{ }^{+}$state; see Fig. 3a, b). Afterward, when we impose a large negative field $E=-4 \mathrm{kV} / \mathrm{cm}$ on the positively poled substrate, the polarization switching pathways include $71^{\circ}$ $\left(180^{\circ}\right)$ ferroelectric domain switching from $P_{r}^{+}$to $P_{3}^{-}\left(P_{1}^{-}\right)$ (see Fig. 3a), and $109^{\circ}$ ferroelastic domain switching from $P_{r}^{+}$to $P_{2}^{-}$(denoted the $P_{r}^{-}$state; see Fig. $3 \mathrm{~b}$ ). The in-plane projections of polarization vectors (i.e., $P^{+}$and $P^{-}$in the (001) plane) are parallel before and after $71^{\circ} / 180^{\circ}$ domain switching (Fig. 3c), while they are perpendicular before and after $109^{\circ}$ domain switching (Fig. 3d). Therefore, the piezostrain effect induced by $71^{\circ} / 180^{\circ}$ ferroelectric domain switching is responsible for the volatile butterfly-like part of the electric field control of the magnetism in Fig. 2c. In contrast to $71^{\circ} / 180^{\circ}$ switching, $109^{\circ}$ ferroelastic domain switching is accompanied by a unique in-plane shear strain in the PMN-PT substrate ${ }^{1,39}$. Based on the lattice parameters $\left(a=4.017 \AA, \theta=89.89^{\circ}\right)^{40}$ of rhombohedral PMNPT, the in-plane shear strain can be estimated to be $\sim 0.19 \%$. In this scenario, two in-plane strain states are generated after removing the field (reducing $E= \pm 4 \mathrm{kV} / \mathrm{cm}$ to $\pm 0 \mathrm{kV} /$ $\mathrm{cm}$ ) because of the stability of the remnant out-of-plane (upward and downward) polarization vectors of PMN-PT. Accordingly, two distinct stable and nonvolatile magnetic states ( $\boldsymbol{A}$ and $\boldsymbol{B}$ states) can be realized through the electrically controlled ferroelastic shear strain effect, as also evidenced by the change in magnetic hysteresis loops for the $P_{r}^{+}$and $P_{r}^{-}$states measured via VSM. The converse ME coupling coefficient equals $\alpha=\mu_{0} \Delta M_{r} / \Delta E=7.37 \times 10^{-8} \mathrm{~s}$ $\mathrm{m}^{-1}$, which is much larger than that for a single-phase 


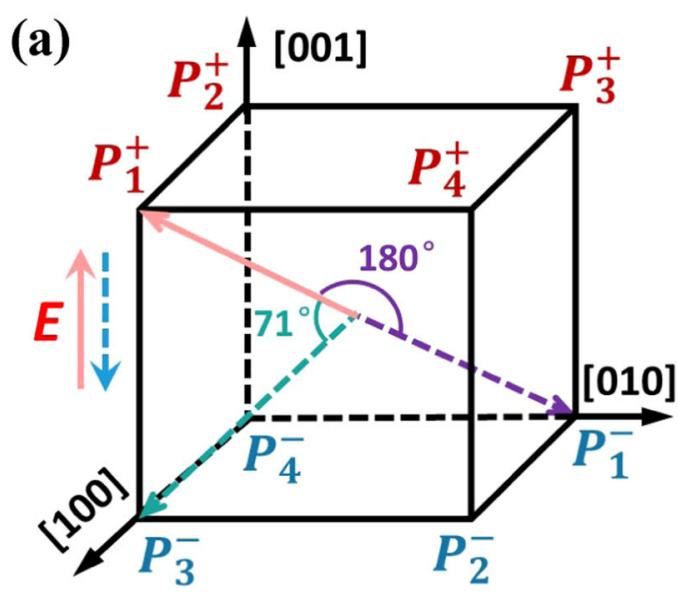

(c)

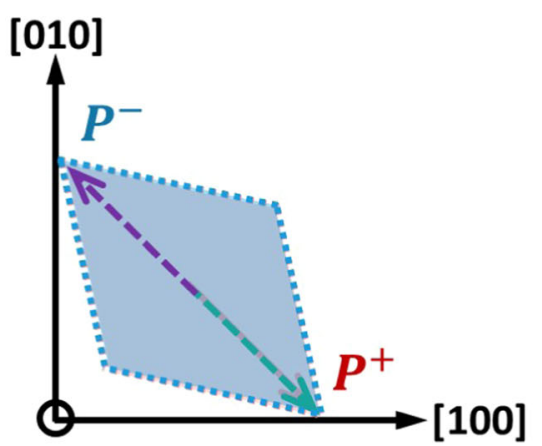

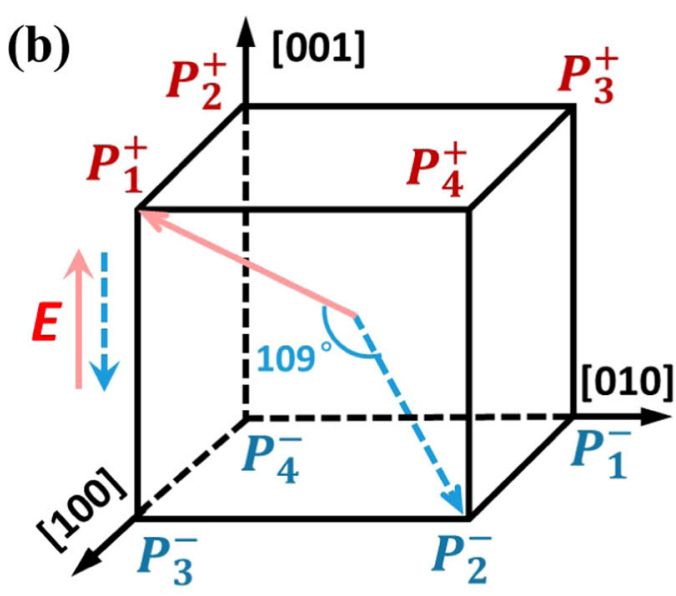

(d)

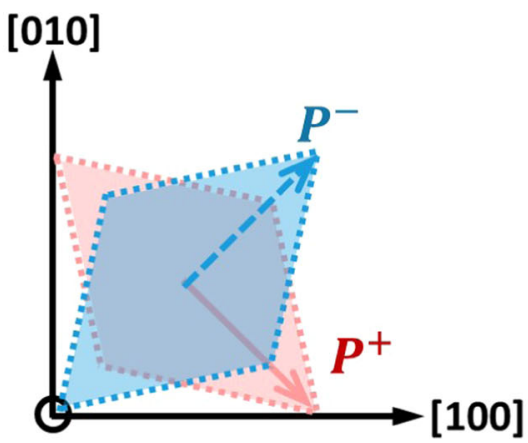

Fig. 3 The switching paths of three kinds of ferroelectric domain switching for the PMN-PT substrate. Schematic diagrams of domain structures for the PMN-PT substrate from the $P_{r}^{+}$to $P_{r}^{-}$states after $\mathbf{a} 71^{\circ} / 180^{\circ}$ domain switching and $\mathbf{b} 109^{\circ}$ domain switching. $\mathbf{c}, \mathbf{d}$ Corresponding in-plane projections $\left(P^{+}\right.$and $\left.P^{-}\right)$of polarization vectors.

multiferroic material $\left(\alpha=3 \times 10^{-10} \mathrm{~s} \mathrm{~m}^{-1} \text { in } \mathrm{TbPO}_{4}\right)^{41}$ and comparable to that for a composite system $\left(\alpha \leq 6 \times 10^{-8} \mathrm{~s}\right.$ $\mathrm{m}^{-1}$ in $\left.\mathrm{La}_{0.7} \mathrm{Sr}_{0.3} \mathrm{MnO}_{3} / \mathrm{PMN}-\mathrm{PT}\right)^{42}$. Similar ME hysteresis responses were also observed in other multiferroic heterostructures, such as the $\mathrm{CoFeB} / \mathrm{PMN}-\mathrm{PT}^{37}, \mathrm{Fe} / \mathrm{PMN} \mathrm{PT}^{36}$, and $\mathrm{MnPt} / \mathrm{PMN}-\mathrm{PT}^{39}$ systems. Our finding discloses that the nonvolatile converse ME effect can be achieved not only in amorphous ${ }^{37}$ and polycrystalline samples ${ }^{36,39}$ but also in epitaxial ferromagnetic thin films.

In addition to static magnetic properties, the magnetization dynamics in multiferroic heterostructures can also be moderately affected by an external electric stimulus through strain-mediated magnetoelectric coupling $^{11}$. Figure $4 \mathrm{a}$, b shows the color-coded transmission parameter $S_{21}$ of the FeRh thin film as a function of microwave frequency and magnetic bias field, when the PMN-PT substrate is in the $P_{r}^{+}$and $P_{r}^{-}$states, respectively, as measured using the experimental setup in Fig. 4c. Obviously, the main absorption peak in the $S_{21}$ spectra arising from the FMR strongly depends on the polarization state of PMN-PT. With the polarization switching from the $P_{r}^{+}$to $P_{r}^{-}$state, the FMR frequency of the FeRh film is visibly enhanced, especially for the low magnetic field region. Another noteworthy feature is that for a specific low frequency, two FMR peaks appear, which is independent of the electric field polarity. The FeRh film exhibits a direct proportionality of the FMR frequency to the magnetic field above a threshold field, where the magnetization is fully aligned with the applied field. Below the threshold (i.e., the alignment field), the resonance frequency decreases with increasing magnetic field since the magnetization is not fully aligned ${ }^{43,44}$.

To further explore the electric field effect on the FMR behavior, the $S_{21}$ spectra of the FeRh film taken in fieldsweeping mode at select frequencies for the $P_{r}^{+}$and $P_{r}^{-}$states of the PMN-PT substrate are illustrated in Fig. 5a-c. Two FMR absorption peaks are observed under microwave excitation at $f=9.55 \mathrm{GHz}$ for the $P_{r}^{+}$or $P_{r}^{-} \quad$ state, which can be separately fitted by Lorentzian functions $S_{21}=C_{\text {sym }} \times \frac{(\Delta H)^{2}}{(\Delta H)^{2}+\left(H-H_{\text {res }}\right)^{2}}+$ $C_{\text {sym }} \times \frac{\Delta H\left(H-H_{\text {res }}\right)}{(\Delta H)^{2}+\left(H-H_{\text {res }}\right)^{2}}+B$ (ref. $\left.{ }^{43}\right)$, where $H_{\text {res }}$ is the peak resonance field, $\Delta H$ is the linewidth, $H$ is the applied magnetic field, $C_{\text {sym }}$ and $C_{\text {asym }}$ are the symmetric and antisymmetric Lorentzian coefficients, and $B$ is the 

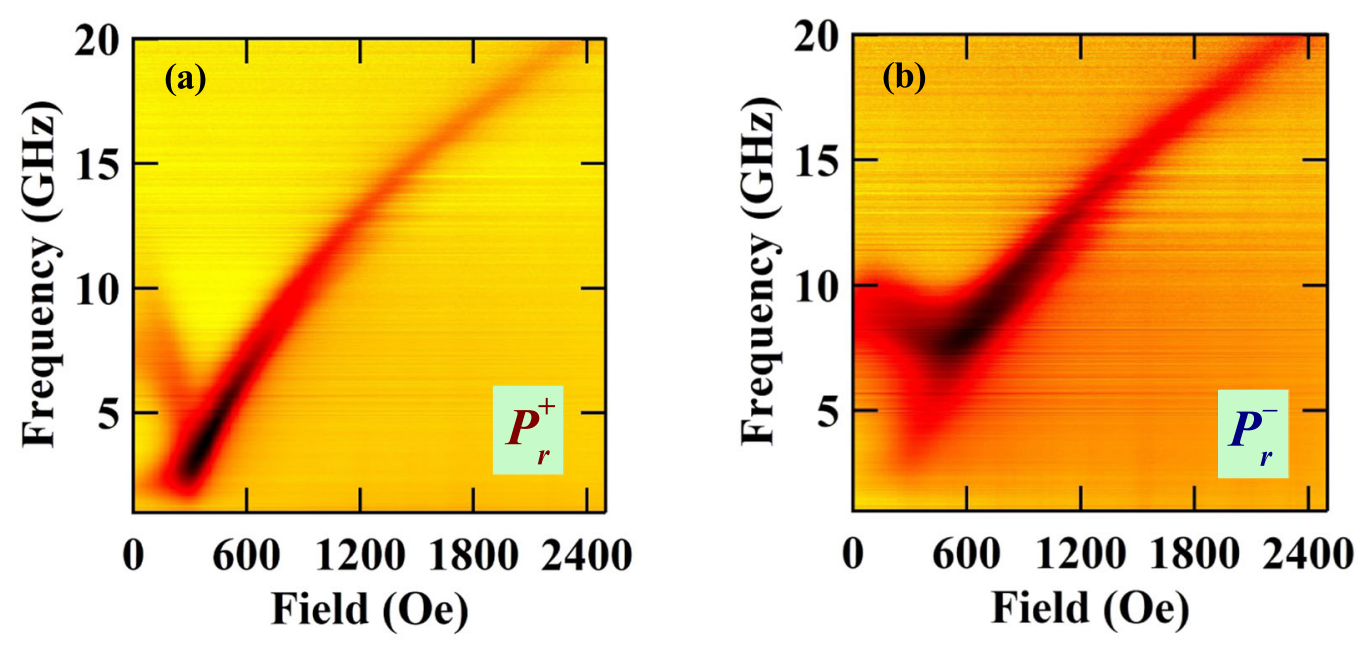

(c)
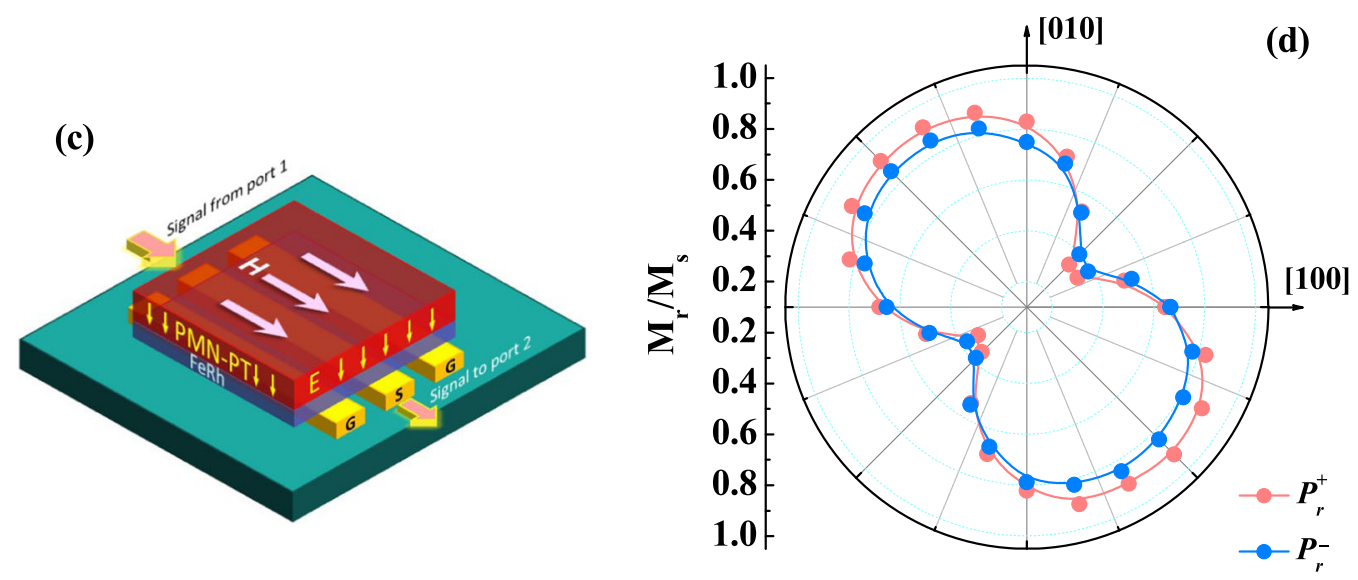

Fig. 4 Electric field dependence of FMR and remnant magnetization ratio in FeRh/PMN-PT structure. $\mathbf{a}$, $\mathbf{b}$ Color-coded transmission parameter $S_{21}$ of the FeRh/PMN-PT structure as a function of microwave frequency and magnetic bias field in the $P_{r}^{+}$and $P_{r}^{-}$states, respectively. c Schematic of FMR measurement. $\mathbf{d}$ Angular dependence of the remnant magnetization ratio (i.e., $M_{r} / M_{s}$ versus $\theta$ curve) of the FeRh/PMN-PT structure in the $P_{r}^{+}$and $P_{r}^{-}$states.

spectral background. As $f$ decreases to $7.65 \mathrm{GHz}$, associated with the polarization switching from the $P_{r}^{+}$to $P_{r}^{-}$ state, the smaller resonance peak at a low magnetic field disappears. This phenomenon indicates that the electricfield-generated shear strain causes a significant change in magnetic anisotropy for the FeRh film, which makes the magnetization fully aligned with the applied field even below the threshold field for the $P_{r}^{-}$state. For $f=$ $3.85 \mathrm{GHz}$, there is only one resonance peak for both the $P_{r}^{+}$and $P_{r}^{-}$states with a large resonance field shift of | $\Delta H_{\text {res }} \mid=111$ Oe. Such remarkable FMR tuning (including the number and location of resonance peaks) by shear strain is believed to be unprecedented and implies the possibility that our FeRh/PMN-PT structures can be used as highly sensitive mechanics-magnetic sensing systems. To explore the link between the FMR effect and the electric-field-induced magnetic anisotropy (i.e., magnetoelastic anisotropy), the resonance frequency is plotted against the resonance field for the $P_{r}^{+}$and $P_{r}^{-}$states in Fig. 5d. In the high-field region, the dispersion relationship can be fitted using the Kittel equation $\left.f=\frac{\gamma}{2 \pi} \sqrt{\left(H+H_{k}\right)\left(H+H_{k}+4 \pi M_{s}\right)}\right)^{11}$, where $\gamma$ is the gyromagnetic ratio, $H_{k}$ is the electric-field-induced effective magnetic field, and $4 \pi M_{s}$ is the magnetization. Based on the fitting results, we obtained an anisotropy field shift of $\Delta H_{k}=130$ Oe when the polarization evolves from the $P_{r}^{+}$to $P_{r}^{-}$state. The electrically induced enhancement of $H_{k}$ will lead to a decrease (increase) in $H_{\text {res }}\left(f_{\text {res }}\right)$ for a fixed frequency (field), which is in accordance with the singlefrequency FMR measurements in Fig. 5a-c. For example, we obtain $\Delta H_{\text {res }}=-120 \mathrm{Oe}$ at $f=2.9 \mathrm{GHz}$ and $\Delta f_{\text {res }}=$ $1.9 \mathrm{GHz}$ at $H=313 \mathrm{Oe}$, corresponding to microwave $\mathrm{ME}$ coupling coefficients of $15 \mathrm{Oecm} / \mathrm{kV}(\Delta H / \Delta \mathrm{E})$ and $237 \mathrm{MHz} \mathrm{cm} / \mathrm{kV}(\Delta f / \Delta \mathrm{E})$, respectively. In the low-field region, the FMR frequency can be described by the equation $f=\frac{\gamma}{2 \pi} \sqrt{\left(H_{k}+4 \pi M_{s}\right)^{2}-H^{2}}$ (ref. $\left.{ }^{43}\right)$. The 

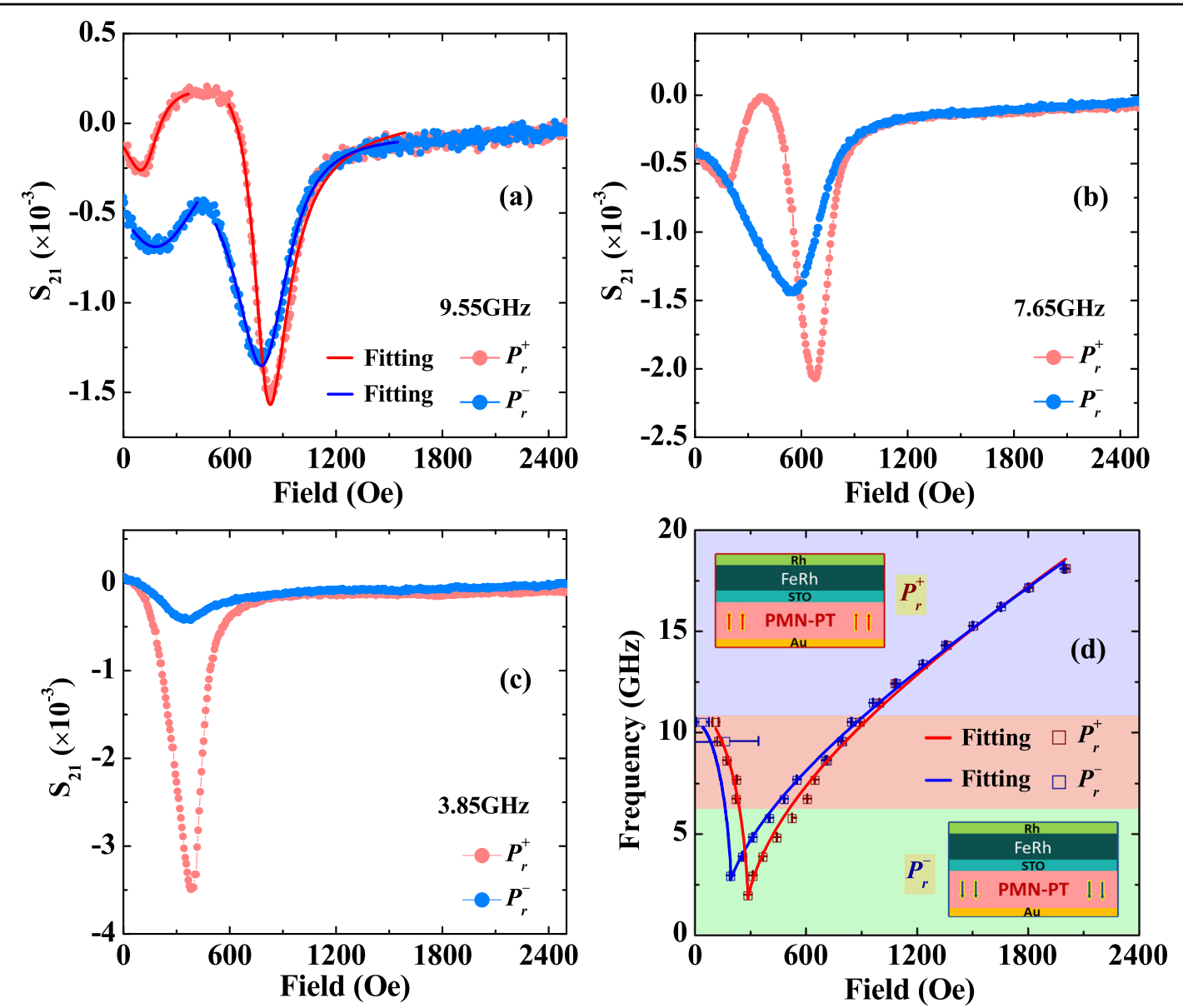

Fig. 5 Electric field dependence of resonance field at various microwave frequencies in FeRh/PMN-PT structure. a-c $S_{21}$ spectra of the FeRh film taken in field-sweeping mode at select frequencies for the $P_{r}^{+}$and $P_{r}^{-}$states of the PMN-PT substrate. Red and blue solid lines in a are the fitting results using Lorentzian functions. $\mathbf{d}$ Resonance frequency as a function of the resonance field of the structure for the $P_{r}^{+}$and $P_{r}^{-}$states. Red and blue solid lines are the fitting curves. Insets show schematics of the multiferroic heterostructure with different polarization directions.

electric-field-induced anisotropy field shift can be determined to be $\Delta H_{k}=125 \mathrm{Oe}$, which is similar to the fitting result obtained using the Kittel equation for the high-field branch. To validate the in-plane magnetic anisotropy and electric-field-induced change, we measured the angulardependent remnant magnetization ratio (i.e., $M_{r} / M_{s}$ versus $\theta$ curve) of the FeRh/PMN-PT structure in the $P_{r}^{+}$and $P_{r}^{-}$states, as shown in Fig. $4 \mathrm{~d}$. The $M_{r} / M_{s}-\theta$ curves for the $P_{r}^{+}$and $P_{r}^{-}$states do not coincide, which demonstrates the strong dependence of magnetic properties on the polarization state and agrees with the nonvolatile converse ME coupling in Fig. 2b. Notably, the sample exhibits a dumbbell-like twofold anisotropy with the magnetic easy axis along the $[-110]$ direction and hard axis along the [110] direction. Upon polarization switching from the $P_{r}^{+}$ to $P_{r}^{-}$state, the twofold anisotropy decreases slightly. This suggests that a small fraction of remnant magnetization is rotated from the $[-110]$ to $[110]$ direction, which can be interpreted in terms of the competition between the magnetocrystalline anisotropy energy and the uniaxial anisotropy energy, as reported in the FeSi/PMN-PT and Fe/PMN-PT systems ${ }^{8,36}$. Therefore, the electrically driven FMR effect is a consequence of shear-strain-mediated magnetoelastic coupling through alteration of the in-plane uniaxial magnetic anisotropy. All these results explicitly establish the effectiveness of shear strain control of both static and dynamic magnetic responses of epitaxial ferromagnetic thin films by simply varying the polarity of the external electric field.

It is attractive to compare the FeRh/PMN-PT structure with the $\mathrm{Fe}_{50} \mathrm{Rh}_{50} / \mathrm{BaTiO}_{3}$ structure in a recent report ${ }^{4}$. Both structures are capable of realizing precise finetuning of magnetic responses by exerting an electric stimulus because of the efficient and continuous strain 
transfer from the ferroelectric substrates to the overlying thin films. In the case of using $\mathrm{BaTiO}_{3}$ crystals as substrates, the origin of the ME effect is the strain-mediated transition between antiferromagnetic and ferromagnetic order in the $\mathrm{Fe}_{50} \mathrm{Rh}_{50}$ films, which is reminiscent of the electroresistance effect in phase-separated perovskite manganite/PMN-PT systems $^{30-33}$. Moreover, upon removal of the electric field, the magnetic properties decay to the initial states due to the linear piezoresponse of the $\mathrm{BaTiO}_{3}$ substrate. In this case, the electrically controlled FMR is ascribed to strain-induced magnetoelastic coupling. By taking advantage of the ferroelastic shear strain effect of PMN-PT, nonvolatile electric field tuning of the magnetism can be achieved by a voltage impulse instead of a voltage bias, which can further reduce power consumption in ME devices. In particular, it has been reported that the spin structure and anomalous Hall effect can be strongly modified by an electric field in antiferromagnetic intermetallic alloys, such as $\mathrm{Fe}_{50} \mathrm{Rh}_{50}$, $\mathrm{Mn}_{3} \mathrm{Pt}, \mathrm{Mn}_{3} \mathrm{Sn}$, and $\mathrm{Mn}_{3} \mathrm{Ga}^{45,46}$. We thus expect that by integrating these alloy films with STO-buffered PMN-PT (001) single crystals, shear strain control of the nonvolatile magnetic phase transition, antiferromagnetic resonance $(\mathrm{THz})$ and Hall resistance switching can be realized for high-density antiferromagnetic spintronic device applications.

\section{Conclusions}

In summary, we have reported nonvolatile electric field control of FMR using an epitaxial FeRh/PMN-PT multiferroic heterostructure. We attribute this to the electrically produced stable and nonvolatile in-plane shear strain state in the PMN-PT substrate, which can modify the in-plane uniaxial magnetic anisotropy of the film. The strong dependence of the resonance frequency/field on the electric field impulse can be manifested by the shearstrain-induced disappearance of the resonance peak in a low field upon polarization switching. Furthermore, by using in situ Kerr measurements, we demonstrate that it is electric-field-induced $109^{\circ}$ ferroelastic domain switching, not $71^{\circ} / 180^{\circ}$ ferroelectric domain switching, that causes the shear strain state and dominates the nonvolatile magnetic change. Our findings are instructive for realizing ferroelastically driven nonvolatile manipulation of latticecoupled static and dynamic magnetic responses in hybrid metal/perovskite systems and for designing nextgeneration reconfigurable, high-frequency, ultralowpower nonvolatile ME, and spintronic devices. Such a hitherto unappreciated approach using shear strain engineering can be extended to develop a rich variety of functional thin film/ferroelectric systems for future information storage and processing.

\section{Acknowledgements}

This work is supported by the National Natural Science Foundation of China (Grant No. 12004423), the Natural Science Foundation of Jiangsu Province, China (Grant No. BK20200662), and the Program for High-Level Entrepreneurial and Innovative Talents Introduction of Jiangsu Province and the High-End Talents Program of China University of Mining and Technology (Grant No. 102520057). This work is also supported by JST CREST (Grant No. JPMJCR18J1) JSPS KAKENHI (Grant Nos. JP17H03377, JP18F18353, and JP17J08317), the Asahi Glass Foundation, and the Kato Foundation for Promotion of Science. M.Z. is thankful for the JSPS Fellowships for Research in Japan.

\section{Author details}

${ }^{1}$ School of Materials Science and Physics, China University of Mining and Technology, 221116 Xuzhou, China. ${ }^{2}$ Department of Physics, Nagoya University, Furo-cho, Chikusa-ku, Nagoya

464-8602, Japan. ${ }^{3}$ Center for Spintronics Research Network, Graduate School of Engineering Science, Osaka University, Toyonaka, Osaka 560-8531, Japan

\section{Conflict of interest}

The authors declare that they have no conflict of interest.

\section{Publisher's note}

Springer Nature remains neutral with regard to jurisdictional claims in published maps and institutional affiliations.

Received: 28 October 2020 Revised: 1 December 2020 Accepted: 9 December 2020.

Published online: 22 January 2021

\section{References}

1. Ghidini, M. et al. Shear-strain-mediated magnetoelectric effects revealed by imaging. Nat. Mater. 18, 840 (2019).

2. Yan, $\mathrm{H}$. et al. A piezoelectric, strain-controlled antiferromagnetic memory insensitive to magnetic fields. Nat. Nanotechnol. 14, 131 (2019).

3. Liu, Z. Q. et al. Electrical switching of the topological anomalous Hall effect in a non-collinear antiferromagnet above room temperature. Nat. Electron. 1, 172 (2018).

4. Cherifil, R. O. et al. Electric-field control of magnetic order above room temperature. Nat. Mater. 13, 345 (2014).

5. Taniyama, T. Electric-field control of magnetism via strain transfer across ferromagnetic/ferroelectric interfaces. J. Phys. Cond. Matter 27, 504001 (2015).

6. Franke, K. J. A. et al. Reversible electric-field driven magnetic domain wall motion. Phys. Rev. X 5, 0110101 (2015).

7. Shirahata, Y. et al. Electric-field switching of perpendicularly magnetized multilayers. NPG Asia Mater. 7, e198 (2015).

8. Guo, X. et al. Electric field induced magnetic anisotropy transition from fourfold to twofold symmetry in (001) $0.68 \mathrm{~Pb}\left(\mathrm{Mg}_{1 / 3} \mathrm{Nb}_{2 / 3}\right) \mathrm{O}_{3}-0.32 \mathrm{PbTiO}_{3} /$ $\mathrm{Fe}_{0.86} \mathrm{Si}_{0.14}$ epitaxial heterostructures. Appl. Phys. Lett. 108, 152401 (2016).

9. Guo, E. J. et al. Tuning the switching time of $\mathrm{BiFeO}_{3}$ capacitors by electrodes' conductivity. Appl. Phys. Lett. 103, 022905 (2013).

10. Wu, S. Z. et al. Strain-mediated electric-field control of exchange bias in a $\mathrm{CO}_{90} \mathrm{Fe}_{10} / \mathrm{BiFeO}_{3} / \mathrm{SrRuO}_{3} / \mathrm{PMN}$-PT heterostructure. Sci. Rep. 5, 8905 (2015).

11. Liu, M. et al. Voltage tuning of ferromagnetic resonance with bistable magnetization switching in energy-efficient magnetoelectric composites. Adv. Mater. 25, 1435 (2013)

12. Yang, Y. J. et al. Controlling the anomalous Hall effect by electric-field-induced piezo-strain in $\mathrm{Fe}_{40} \mathrm{Pt}_{60}$ (001)- $\mathrm{Pb}\left(\mathrm{Mg}_{1 / 3} \mathrm{Nb}_{2 / 3}\right)_{0.67} \mathrm{Ti}_{0.33} \mathrm{O}_{3}$ multiferroic heterostructures. Appl. Phys. Lett. 112, 033506 (2018).

13. Okabayashi, J., Miura, Y. \& Taniyama, T. Strain-induced reversible manipulation of orbital magnetic moments in $\mathrm{Ni} / \mathrm{Cu}$ multilayers on ferroelectric $\mathrm{BaTiO}_{3}$. npj Quantum Mater. 4, 21 (2019).

14. Suzuki, I., Itoh, M. \& Taniyama, T. Elastically controlled magnetic phase transition in $\mathrm{Ga}-\mathrm{FeRh} / \mathrm{BaTiO}_{3}(001)$ heterostructure. Appl. Phys. Lett. 104, 022401 (2014).

15. Sheng, Z. G., Gao, J. \& Sun, Y. P. Coaction of electric field induced strain and polarization effects in $\mathrm{La}_{0.7} \mathrm{Ca}_{0.3} \mathrm{MnO}_{3} / \mathrm{PMN}-\mathrm{PT}$ structures. Phys. Rev. B 79, 174437 (2009). 
16. Gou, J. et al. Ferromagnetic composite with stress-insensitive magnetic permeability: compensation of stress induced anisotropies. Phys. Rev. Mater. 2 114406 (2018).

17. Gao, W. X. et al. Flexible organic ferroelectric films with a large piezoelectric response. NPG Asia Mater. 7, e189 (2015).

18. Zhu, Q. X. et al. Ultrahigh tunability of room temperature electronic transport and ferromagnetism in dilute magnetic semiconductor and PMN-PT singlecrystal-based field effect transistors via electric charge mediation. Adv. Funct. Mater. 25, 1111 (2015)

19. Molegraaf, H. J. A. et al. Magnetoelectric effects in complex oxides with competing ground states. Adv. Mater. 21, 3470 (2009).

20. $W u, T$. et al. Domain engineered switchable strain states in ferroelectric (011) $\left[\mathrm{Pb}\left(\mathrm{Mg}_{1 / 3} \mathrm{Nb}_{2 / 3}\right) \mathrm{O}_{3}\right](1-\mathrm{x})-\left[\mathrm{PbTiO}_{3}\right] \times(\mathrm{PMN}-\mathrm{PT}, \mathrm{x} \approx 0.32)$ single crystals. J. Appl. Phys. 109, 124101 (2011).

21. Wu, T. et al. Giant electric-field-induced reversible and permanent magnetization reorientation on magnetoelectric $\mathrm{Ni} / \mathrm{(011)}\left[\mathrm{Pb}\left(\mathrm{Mg}_{1 / 3} \mathrm{Nb}_{2 / 3}\right) \mathrm{O}_{3}\right](1-\mathrm{x})-$ $\left[\mathrm{PbTiO}_{3}\right] \times$ heterostructure. Appl. Phys. Lett. 98, 012504 (2011).

22. Liu, M. et al. Non-volatile ferroelastic switching of the Verwey transition and resistivity of epitaxial $\mathrm{Fe}_{3} \mathrm{O}_{4} / \mathrm{PMN}-\mathrm{PT}$ (011). Sci. Rep. 3, 1876 (2013).

23. Zheng, M., Sun, H. L., Chan, M. K. \& Kwok, K. W. Reversible and nonvolatile tuning of photoluminescence response by electric field for reconfigurable luminescent memory devices. Nano Energy 55, 22 (2019).

24. Yang, M. M. et al. Intrinsic and quantitative effects of in-plane strain on ferroelectric properties of $\mathrm{Mn}$-doped $\mathrm{BiFeO}_{3}$ epitaxial films by in situ inducing strain in substrates. Appl. Phys. Lett. 104, 052902 (2014).

25. Zhi, B. W. et al. Electric-field-modulated nonvolatile resistance switching in VO2/PMN-PT(111) heterostructures. ACS Appl. Mater. Interfaces 6, 4603 (2014).

26. Nan, T. X., Liu, M., Ren, W., Ye, Z. G. \& Sun, N. X. Voltage control of metalinsulator transition and non-volatile ferroelastic switching of resistance in VOX PMN-PT heterostructures. Sci. Rep. 4, 5931 (2014).

27. Salev, P., Valle, J., del, Kalcheim, Y. \& Schuller, I. K. Giant nonvolatile resistive switching in a Mott oxide and ferroelectric hybrid. Proc. Natl Acad. Sci. USA 116, 8798 (2019).

28. Yan, J. et al. Manipulation of the electronic transport properties of chargetransfer oxide thin films of $\mathrm{NdNiO}_{3}$ using static and electric-field-controllable dynamic lattice strain. Phys. Rev. Appl. 11, 034037 (2019).

29. Zheng, M. et al. Ferroelastic strain control of multiple nonvolatile resistance tuning in $\mathrm{SrRuO}_{3} / \mathrm{PMN}-\mathrm{PT}(111)$ multiferroic heterostructures. Appl. Phys. Lett. 110, 182403 (2017).

30. Zheng, $M$. et al. Tunable interface strain coupling and its impact on the electronic transport and magnetic properties of $\mathrm{La}_{05} \mathrm{Ca}_{05} \mathrm{MnO}_{3} / \mathrm{Pb}\left(\mathrm{In}_{1 / 2} \mathrm{Nb}_{1 / 2}\right)$ $\mathrm{O}_{3}-\mathrm{Pb}\left(\mathrm{Mg}_{1 / 3} \mathrm{Nb}_{2 / 3}\right) \mathrm{O}_{3}-\mathrm{PbTiO}_{3}$ multiferroic heterostructures. Phys. Rev. B 90, 224420 (2014)
31. Zheng, M. \& Zheng, R. K. Electric-field-tunable ferroelastic control of nonvolatile resistivity and ferromagnetic switching in multiferroic $\mathrm{La}_{0.67} \mathrm{Ca}_{0.33} \mathrm{MnO}_{3} /\left[\mathrm{PbMg}_{1 / 3} \mathrm{Nb}_{2 / 3} \mathrm{O}_{3}\right]_{0.7}\left[\mathrm{PbTiO}_{3}\right]_{0.3}$ heterostructures. Phys. Rev. Appl. 5, 044002 (2016).

32. Zheng, M. et al. Optically tunable resistive-switching memory in multiferroic heterostructures. Phys. Rev. Appl. 9, 044039 (2018).

33. Zheng, M. et al. Ferroelastically and magnetically co-coupled resistive switching in $\mathrm{Nd}_{0.5} \mathrm{Sr}_{0.5} \mathrm{MnO}_{3} / \mathrm{PMN}-\mathrm{PT}(011)$ multiferroic heterostructures. Appl. Phys. Lett. 112, 123502 (2018).

34. Swartzendruber, L. J. The Fe-Rh (Iron-Rhodium) system. Bull. Alloy Phase Diagr 5, 456 (1984).

35. Usami, T., Itoh, M. \& Taniyama, T. Compositional dependence of Gilbert damping constant of epitaxial $\mathrm{Fe}_{100-x} \mathrm{Rh} \times \mathrm{x}$ thin films. Appl. Phys. Lett. 115, 142403 (2019).

36. Zhang, S. et al. Strain-mediated coexistence of volatile and nonvolatile converse magnetoelectric effects in $\mathrm{Fe} / \mathrm{Pb}\left(\mathrm{Mg}_{1 / 3} \mathrm{Nb}_{2 / 3}\right)_{0.7} \mathrm{Ti}_{0.3} \mathrm{O}_{3}$ heterostructure. ACS Appl. Mater. Interfaces 9, 20637 (2017).

37. $\mathrm{Ba}, \mathrm{Y}$. et al. Spatially resolved electric-field manipulation of magnetism for CoFeB mesoscopic discs on ferroelectrics. Adv. Funct. Mater. 28, 1706448 (2018).

38. Zheng, M. et al. Optically and electrically co-controlled resistance switching in complex oxide heterostructures. Appl. Phys. Lett. 111 172901 (2017)

39. Liu, Z. Q. et al. Electrically reversible cracks in an intermetallic film controlled by an electric field. Nat. Commun. 9, 41 (2018).

40. Noheda, B., Cox, D. E., Shirane, G., Gao, J. \& Ye, Z. G. Phase diagram of the ferroelectric relaxor (1-x) $\mathrm{PbMg}_{1 / 3} \mathrm{Nb}_{2 / 3} \mathrm{O}_{3}-\mathrm{xPbTiO}_{3}$. Phys. Rev. B 66, 054104 (2002).

41. Rado, G. T., Ferrari, J. M. \& Maisch, W. G. Magnetoelectric susceptibility and magnetic symmetry of magnetoelectrically annealed $\mathrm{TbPO}_{4}$. Phys. Rev. B 29 4041 (1984).

42. Thiele, C., Dörr, K., Bilani, O., Rödel, J. \& Schultz, L. Influence of strain on the magnetization and magnetoelectric effect in $\mathrm{La}_{07} \mathrm{~A}_{03} \mathrm{MnO}_{3} / \mathrm{PMN}-\mathrm{PT}(001)$ $(A=S r, C a)$. Phys. Rev. B 75, 054408 (2007).

43. He, C. et al. Spin-torque ferromagnetic resonance in W/Co-Fe-B/W/Co-Fe-B/ MgO stacks. Phys. Rev. Appl. 10, 034067 (2018).

44. Montoya, E. et al. in Handbook of Surface Science, Vol. 5, Ch. 3 113-167 (Elsevier, North-Holland, 2015).

45. Yan, $\mathrm{H}$. et al. Electric-field-controlled antiferromagnetic spintronic devices. $A d v$. Mater. 32, 1905603 (2020).

46. Guo, H. et al. Giant piezospintronic effect in a noncollinear antiferromagnetic metal. Adv. Mater. 32, 2002300 (2020). 